\title{
latrogenic blood loss due to daily laboratory testing and the risk of subsequent anaemia in intensive care unit patients: case series
}

\author{
Kamil Witosz'1, Olga Wojnarowicz¹ and Łukasz J. Krzych²⿴ \\ 'Students' Scientific Society, Department of Anaesthesiology and Intensive Care, School of Medicine in Katowice, Medical University of Silesia, \\ Katowice, Poland; 'Department of Anaesthesiology and Intensive Care, School of Medicine in Katowice, Medical University of Silesia, Katowice, \\ Poland
}

Introduction: Anaemia is associated with a wide range of negative outcomes. Diagnostic blood loss (DBL) may contribute to its occurrence. We aimed to evaluate DBL and its impact on haemoglobin (HGB) concentration and developing anaemia in the intensive care unit (ICU) patients. Methods: A study group comprised of 36 adult ICU patients. DBL during 7 consecutive, postadmission days was calculated. Anaemia occurrence was assessed using the WHO thresholds. Data on HGB and haematocrit (HCT) was subjected to analysis. Results: Upon admission, 24 (67\%) patients were diagnosed with anaemia, on the eighth day $29(80 \%)$ subjects (with 6 new cases). The median volume of blood collected was $143.15 \mathrm{~mL}$ (IQR 121.4-161.65) per week. No differences in DBL were found between the subjects with newly developed anaemia and their counterparts $(p=0.4)$. The median drop of HGB $(\mathrm{Hb} \Delta)$ was $18 \mathrm{gL}^{-1}$ (IQR 5-28) and the median drop of haematocrit $(\mathrm{Ht} \Delta)$ was $4.55 \%$ (IQR $1.1-7.95)$. There was no correlation between neither $\mathrm{Hb} \Delta$ and $\mathrm{DBL}(p=0.8)$ nor $\mathrm{Ht} \Delta$ and DBL $(p=0.7)$. There were also no differences in $\mathrm{Hb} \Delta / \mathrm{Ht} \Delta$ when age, gender or the primary critical illness were taken into account for the analysis ( $p>0.05$ for all). The 7-day fluid balance was associated with haemoglobin drop ( $R=0.45 ; p=0.006)$. Conclusions: Anaemia is frequent in ICU patients. Diagnostic blood loss in our institution is acceptable and seems to protect patients against significant iatrogenic blood loss and subsequent anaemia. Dilutional anaemia may interfere with the results so before-after interventional research is needed to explore this interesting topic.

Keywords: anaemia, iatrogenic, blood loss, intensive care

Received: 21 October, 2020; revised: 07 January, 2021; accepted: 10 January, 2021; available on-line: 08 March, 2021

$\square$ e-mail: Ikrzych@sum.edu.pl

Abbreviations: APACHE, Acute Physiology and Chronic Health Evaluation; DBL, diagnostic blood loss; $\mathrm{Hb} \Delta$, drop of haemoglobin; $\mathrm{Ht} \Delta$, drop of haematocrit; IQR, interquartile range; ICU, intensive care unit; SAPS, Simplified Acute Physiology Score; SOFA, Sequential Organ Failure Assessment; WHO, World Health Organization

\section{INTRODUCTION}

Anaemia is a common clinical problem in critically ill patients with an upon-admission prevalence reaching about two-thirds of the intensive care unit (ICU) patients (Lasocki et al., 2020). The World Health Organisation (WHO) defines anaemia based on the sex and haemoglobin concentration (HGB) (Blanc et al., 1968) with the value of HGB below $120 \mathrm{gL}^{-1}$ in women and $130 \mathrm{gL}^{-1}$ in men being the cut-off values. Anaemia has a great impact on patients' prognosis, causing increased morbidity, mortality, and hospital length of stay and augmenting the requirement for packed red blood cells (PRBC) transfusions - which are themselves connected to the unfavourable outcome (Maxwell \& Wilson, 2006). The foregoing features are prompting to search for culprits of anaemia and, if possible, to reduce their influence on HBG. The main mechanisms of the anaemia development are blood loss (Pieracci \& Barie, 2006), haemodilution and inflammation (Lasocki et al., 2011). Diagnostic blood loss (DBL) may also contribute to anaemia occurrence. Management and prevention of anaemia in the ICU are difficult, complex and multidirectional (Lasocki et al., 2020). In this study, we aimed to evaluate DBL and its impact on HGB concentration and developing anaemia in patients during the first week of the hospitalization in ICU.

\section{METHODS}

\section{Design and patients}

This case series is based on a prospective, single-centre, observational study of 70 ICU patients treated in the Department of Anaesthesiology and Intensive Care, Medical University of Silesia, Katowice, Poland. The enrolment period was from 25th October 2019 to 12th February 2020. The main inclusion criteria were the age of $\geq 18$ years, at least 8 days of at-ward stay and the presence of an arterial line for the routine blood draw (i.e. in-ward phlebotomy reduction policy). Exclusion criteria included: missing clinical or laboratory data from the first to the eighth day of the ICU stay, suspected or confirmed active bleeding, bleeding as the reason of hospital admission (including bloody fluids drainage, actively bleeding wounds, blood in the stool, melena), undergoing invasive procedures with the risk of substantial bleeding (any open surgery, reoperations, surgical wound treatment, percutaneous tracheostomy). Based on those criteria 34 patients were excluded from the study. None of the patients was diagnosed with haematological malignancy. Three patients had chronic kidney disease at stage 3 .

\section{Data Collection}

Data were collected from hospital charts and electronic medical records, which included basic demo- 
graphics (sex and age), clinical features (diagnosis at admission; renal replacement therapy; iron supplementation; percutaneous gastrostomy; upon-admission assessment of multi-organ failure - Acute Physiology and Chronic Health Evaluation (APACHE) II score, Simplified Acute Physiology Score (SAPS) II, Sequential Organ Failure Assessment (SOFA); laboratory data - HGB, haematocrit (Hct), mean corpuscular volume (MCV), red blood cells (RBC) counts) and volume of blood collected for daily laboratory testing. The weekly decrease (or increase) in $\mathrm{HGB} / \mathrm{Hct} / \mathrm{RBC}$ value was calculated as the difference between the value on the day of admission and that in the morning blood sample on the eighth day of hospital stay.

\section{Definitions}

Anaemia was defined according to the WHO criteria (Blanc et al., 1968). The range of MCV $<80 \mathrm{fL}, 80-100$ $\mathrm{fL}$ and $>100 \mathrm{fL}$ was used to describe anaemia as microcytic, normocytic and macrocytic, respectively (Lasocki et al., 2020).

\section{Diagnostic Blood Loss}

DBL was measured as the total amount of blood collected to test tubes in 7 consecutive days from ICU admission. We recorded the number and types of blood tubes using data from electronic medical records. Each test tube had its specific identity number so we could match a particular laboratory test to every used tube. We had to assume that only the minimal amount of blood needed to run the test was collected and also that there was no additional loss of its volume during the blood draw. From the beginning of this study, we verified the capacity of blood tubes weekly. Arterial blood gas tubes were assigned a volume of $1 \mathrm{~mL}$, haematology tubes $2 \mathrm{~mL}$ and blood cultures were estimated as $10 \mathrm{~mL}$ based on the prior literature (Chant et al., 2006). The volume of coagulation laboratory tubes and chemistry/miscellaneous laboratory tubes had changed during the data collection. Until 24th January 2020, the volume of the former was $1.8 \mathrm{~mL}$ and after that day it was $2.7 \mathrm{~mL}$. The latter was $5 \mathrm{~mL}$ and $2.5 \mathrm{~mL}$ and the change was on 17th January 2020. Due to the fact that all the patients had an arterial line $1.5 \mathrm{~mL}$ of blood needed to be drawn and discarded before each blood sampling (accordingly to the written policy for the arterial line use). That volume, multiplied by the number of blood samplings through the day, was added to the total volume of DBL. A 7-day fluid balance was calculated to assess the extent of dilutional anaemia.

\section{Red blood cell transfusion policy}

Restrictive transfusion strategy (i.e. $\mathrm{HGB}<70 \mathrm{gL}^{-1}$ ) was applied for PRBC transfusions, with possible individual protocol violations due to increased oxygen demand.

\section{Statistical analysis}

Statistical analysis was performed using MedCalc Statistical Software version 17.2 (MedCalc Software bvba, Ostend, Belgium). Continuous variables were expressed as the median and interquartile range (IQR). Qualitative variables were expressed as absolute values. Between-group differences for quantitative variables were assessed using Mann-Whitney U-test, after verification of variables' distribution with Shapiro-Wilk test. The correlation was assessed using the Spearman rank coefficient (R). 'P' value was set at 0.05 .

\section{RESULTS}

After the implementation of the inclusion and exclusion criteria 36 patients were enrolled in this study. Patients characteristic are summarized in Table 1.

Anaemia was diagnosed on admission in $24(67 \%) \mathrm{pa}-$ tients. On the eighth day, there were 29 patients $(80 \%)$ with anaemia, among which there were 6 new cases. The median volume of blood collected was $143.15 \mathrm{~mL}$ (IQR 121.4-161.65) per week. No differences were found in DBL in subjects with newly developed anaemia on day 8 (140.3 mL; 119.6-176.6) and their counterparts (136.4 $\mathrm{mL}$; 108.6-152.5) ( $p=0.4)$ (Fig. 1). APACHE II ( $p=0.6)$, SAPS II $(p=0.4)$ and SOFA $(p=0.7)$ scores were unrelated to anaemia incidence during ICU stay, but there was a significant positive correlation between the volume of collected blood and on-admission assessment of multiorgan dysfunction measured as APACHE II $(\mathrm{R}=0.38$, $p=0.02$; Fig. 2) and SOFA ( $\mathrm{R}=0.60, p<0.001$; Fig. 3). No such correlation was present between DBL and SAPS II $(\mathrm{R}=0.26, p=0.1)$. The median drop of haemoglobin $(\mathrm{Hb} \Delta)$ was $18 \mathrm{gL}^{-1}(5-28)$ and the median drop of haematocrit $(\mathrm{Ht} \Delta)$ was $4.55 \%$ (1.1-7.95). There was no correlation between neither $\mathrm{Hb} \Delta$ and DBL $(p=0.8)$ nor $\mathrm{Ht} \Delta$ and DBL $(p=0.7)$. There were no differences in $\mathrm{Hb} \Delta$ /

Table 1. Demographic and clinical data of enrolled patients

\begin{tabular}{ll}
\hline Variable & Value \\
\hline SEX (M/F) & $21 / 15$ \\
\hline Age, median (IQR) [years] & $63(52.5-70)$ \\
\hline APACHE II, median (IQR) & $18.5(15.5-23)$ \\
\hline SAPS II, median (IQR) & $46(34-55)$ \\
\hline SOFA, median (IQR) & $9(5.5-11)$ \\
\hline HGB, median [gL-1] & \\
\hline On admission (all/M/F) & $117 / 122 / 113$ \\
\hline $8^{\text {th }}$ day (all/M/F) & $95 / 103 / 90$ \\
\hline Ht, median [\%] & \\
\hline On admission (all/M/F) & $35.15 / 36.3 / 34.5$ \\
\hline $8^{\text {th }}$ day (all/M/F) & $30.4 / 32.1 / 28.8$ \\
\hline RBC, median [mln uL-1] & \\
\hline On admission (all/M/F) & $3.82 / 3.94 / 3.74$ \\
\hline $8^{\text {th } ~ d a y ~(a l l / M / F) ~}$ & $3.145 / 3.43 / 2.92$ \\
\hline Anaemia, type [patient] & \\
\hline Microcytic & $22 / 13 / 9$ \\
\hline On admission (all/M/F) & \\
\hline $8^{\text {th }}$ day (all/M/F) & \\
\hline Normocytic & \\
\hline On admission (all/M/F) & \\
\hline $8^{\text {th } ~ d a y ~(a l l / M / F) ~}$ & \\
\hline Macrocytic & \\
\hline On admission (all/M/F) & \\
\hline $8^{\text {th }}$ day (all/M/F) & \\
\hline & \\
\hline
\end{tabular}

Categorical data are depicted as absolute values, continuous data are presented as median with interquartile range (in brackets). 


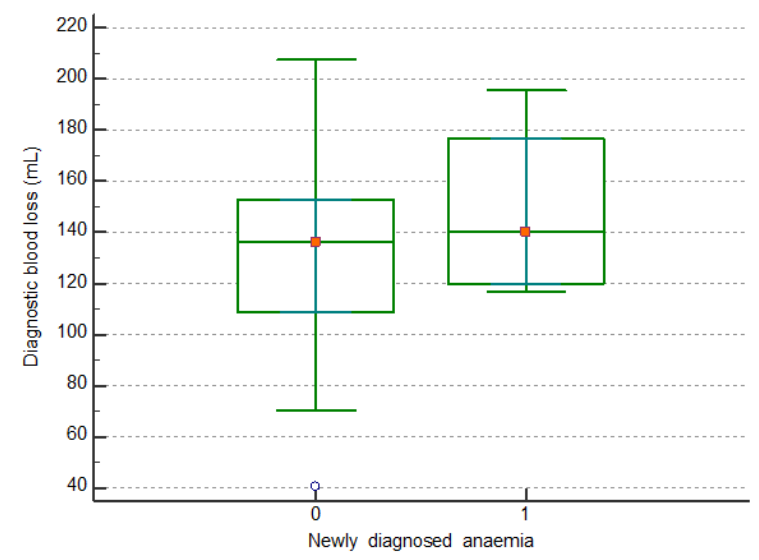

Figure 1. Comparison of diagnostic blood loss in patients with newly developed anaemia and their counterparts

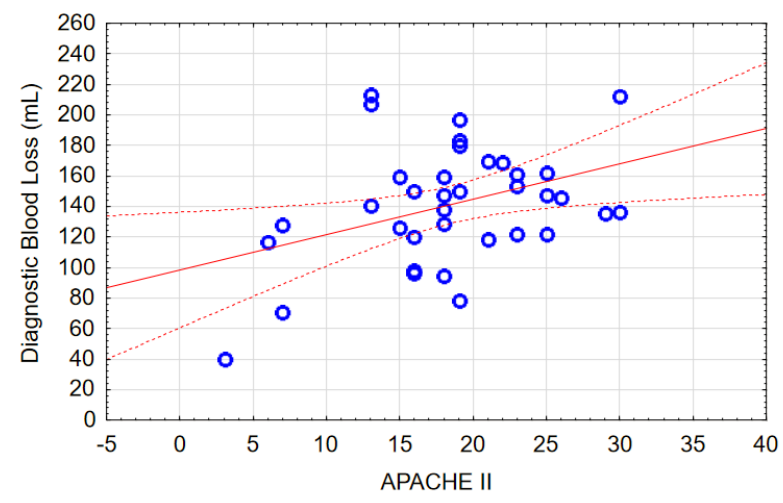

Figure 2. Scatter diagram for diagnostic blood loss and its relation to APACHE II score

Ht $\Delta$ when age, sex or primary critical illness were taken into account for the analysis ( $p>0.05$ for all, data not shown). The median 7-day fluid balance was $-2730 \mathrm{~mL}$ (IQR -8082-550). There was a significant positive correlation between fluid balance and $\mathrm{Hb} \Delta(\mathrm{R}=0.45, p=0.006)$ but no clear association was found for $\mathrm{Ht} \Delta(\mathrm{R}=0.31$, $p=0.07)$. Two of the patients had a packed red blood cell transfusion (1 unit each) which was included in the data (with an HGB trigger of $67 \mathrm{gL}^{-1}$ and $76 \mathrm{gL}^{-1}$ ).

\section{DISCUSSION}

Anaemia is a known factor of increased morbidity and mortality, especially in critically ill patients. Many studies over the years proved that the volume of withdrawn blood can be a strong predictor of the drop in haemoglobin and haematocrit values, contributing to phlebotomy-induced anaemia. The mathematical simulation by Lyon and others (Lyon et al., 2013) proved that the volume of withdrawn blood has a significant influence on the change of HGB values. Also, the study conducted by Thavendiranathan and others (Thavendiranathan et al., 2005) showed that - on average - for every $100 \mathrm{~mL}$ of blood sampling a decrease in haemoglobin and haematocrit can be expected by $7.0 \mathrm{gL}^{-1}$ and $1.9 \%$, respectively.

Unfortunately, anaemia is very common among ICU patients. Among our subjects, 67\% presented on-admission HGB values below the cut-off point for their sex which corresponds with $63 \%$ from a multicentre

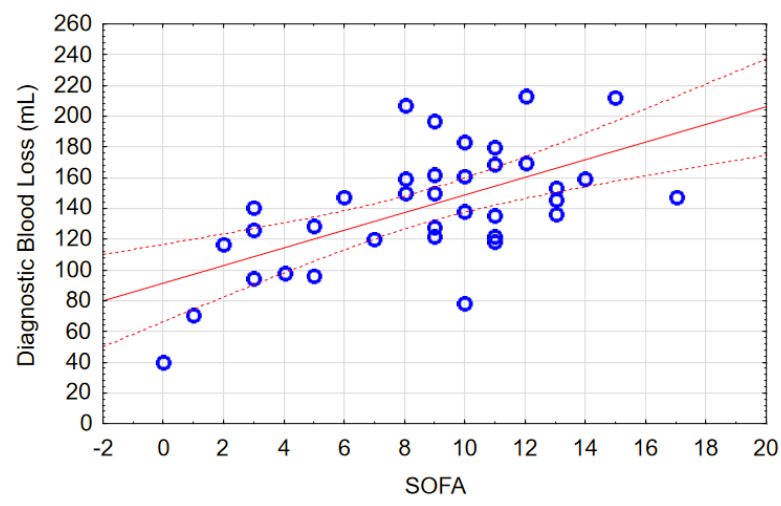

Figure 3. Scatter diagram for diagnostic blood loss and its relation to SOFA score

observational study conducted by Vincent and others (Vincent et al., 2002). By the eighth day, $80 \%$ of them either developed anaemia (6 patients, 17\%) or had a further decrease in HGB value. In only 4 cases the increase of HGB value could have been observed. Although we were unable to confirm that there was a correlation between the volume of the blood draw and the delta of HGB value during a 7-day observation, it appears that it is not without meaning. When taking into account the differences in haemoglobin values between day one and the day on which patient's lowest HGB value was observed, a positive correlation was found $(\mathrm{R}=0.42$, $p=0.01)$. Therefore it is important to conduct therapy according to guidelines aimed to prevent iatrogenic blood loss. Klein and others (Klein et al., 2019) placed great emphasis on the importance of using small (paediatric) tubes for blood sampling to prevent iatrogenic anaemia. Based on the result of their study, Vincent and others (Vincent et al., 2002) concluded that the mean total blood volume (TBV) collected from one ICU patient over 24 hours was $41 \mathrm{~mL}$ (the mean number of samples collected per patient was 4.6). According to Lasocki and others (Lasocki et al., 2020), total blood volume per patient is within the range of 40 to $80 \mathrm{~mL}$ per day. In our study, mean TBV was $18.9 \mathrm{~mL}$ (with 2.1 draws per patient). This visibly smaller TBV was achieved by the common usage of arterial lines and small test tubes (excluding blood culture bottles, no tube allowed to draw more than $5 \mathrm{~mL}$ of blood). These findings are consistent with prior literature (Silver et al., 1993; Sanchez-Giron \& Alvarez-Mora, 2008).

Due to the fact that there is a significant positive correlation between multi-organ dysfunction scores (measured as APACHE II and SOFA) and TBV, prevention of iatrogenic blood loss becomes even more relevant issue. The sicker the patient, the more tests they need, therefore more of their blood is withdrawn. But not all occurrences of anaemia can result from iatrogenic blood loss, as the critical illness itself is a factor of major importance (Baysan et al., 2020).

\section{Study limitations}

Firstly, this research covered 36 patients from a group of 70 subjects hospitalised over the study period. Nevertheless, it was necessary to set up strict exclusion criteria for objective assessment of the impact of DBL on anaemia occurrence. Secondly, a plethora of clinical and iatrogenic factors favour anaemia in the critical care setting. Additional calculations using complex statistical methods are needed to minimise confounding. Thirdly, this re- 
search was observational and interventional study is required to verify our conclusions. On the other hand, this real-life scenario reflects well the blood management policy in our institution. Finally, we had no insight into the risk of pre-hospital bleeding nor the haemostatic status of the patients, which might have interfered with our results (Pereira et al., 2019). We found the risk of dilutional anaemia which might have biased our observations.

\section{CONCLUSIONS}

Anaemia is frequent in ICU patients. Diagnostic blood loss in our institution is acceptable and efforts to protect patients against significant iatrogenic blood loss and subsequent anaemia are effective. Dilutional anaemia may interfere with the results so before-after interventional research is needed to explore this interesting topic.

\section{REFERENCES}

Baysan M, Sesmu Arbous M, van der Bom JG (2020) Iatrogenic anemia: an underestimated and solvable problem in the intensive care unit. Ann. Blood 5: 19. https://doi.org/10.21037/aob-20-29

Blanc B, Finch CA, Hallberg L et al. (1968) Nutritional anaemias. Report of a WHO Scientific Group. WHO Tech. Rep. Ser. 405: 1-40.

Chant C, Wilson G, Friedrich JO (2006) Anemia, transfusion, and phlebotomy practices in critically ill patients with prolonged ICU length of stay: a cohort study. Critical Care 10: R140. https://doi. org/10.1186/cc5054

Klein AA, Bailey CR, Charlton A, Lawson C, Nimmo AF, Payne S, et al. (2019) Association of Anaesthetists: anaesthesia and peri-operative care for Jehovah's Witnesses and patients who refuse blood. Anaesthesia 74: 74-82. https://doi.org/10.1111/anae.14441
Lasocki S, Longrois D, Montravers P, Beaumont C (2011) Hepcidin and anemia of the critically ill patient: bench to bedside. Anesthesiology 114: 688-694. https://doi.org/10.1097/ALN.0b013e3182065c57

Lasocki S, Pène F, Ait-Oufella H et al. (2020) Management and prevention of anemia (acute bleeding excluded) in adult critical care patients. Ann. Intensive Care 10: 97. https://doi.org/10.1186/s13613020-00711-6

Lyon AW, Chin AC, Slotsve GA, Lyon ME (2013) Simulation of repetitive diagnostic blood loss and onset of iatrogenic anemia in critical care patients with a mathematical model. Computers Biol. Med. 43: 84-90. https://doi.org/10.1016/i.compbiomed.2012.11.008

Maxwell MJ, Wilson MJA (2006) Complications of blood transfusion. Continuing Education in Anaesthesia Critical Care \& Pain 6: 225-229. https://doi.org/10.1093/bjaceaccp/mkl053

Pereira B, Dorigatti A, Calderon L, Negrão M, Meirelles G, Duchesne J (2019) Pre-hospital environment bleeding: from history to future prospects. Anaesthesiol Intensive Ther. 51: 240-248. doi: https://doi. org/10.5114/ait.2019.86059

Pieracci FM, Barie PS (2006). Diagnosis and management of iron-related anemias in critical illness. Crit. Care Med. 34: 1898-1905. https:// doi.org/10.1097/01.CCM.0000220495.10510.C1

Sanchez-Giron F, Alvarez-Mora F (2008) Reduction of blood loss from laboratory testing in hospitalized adult patients using smallvolume (pediatric) tubes. Arch. Pathol. Lab. Med. 132: 1916-1919. https://doi.org/10.1043/1543-2165-132.12.1916

Silver MJ, Li YH, Gragg LA, Jubran F, Stoller JK (1993) Reduction of blood loss from diagnostic sampling in critically ill patients using a blood-conserving arterial line system. Chest 104: 1711-1715. https:// doi.org/10.1378/chest.104.6.1711

Thavendiranathan P, Bagai A, Ebidia A, Detsky AS, Choudhry NK (2005) Do blood tests cause anemia in hospitalized patients? The effect of diagnostic phlebotomy on hemoglobin and hematocrit levels. J. Gen. Int. Med. 20: 520-524. https://doi.org/10.1111/j.15251497.2005.0094.x

Vincent JL, Baron JF, Reinhart K, Gattinoni L, Thijs L, Webb A, Meier-Hellmann A, Nollet G, Peres-Bota D; ABC (Anemia and Blood Transfusion in Critical Care) Investigators. JAMA 288: 1499-1507. https://doi.org/10.1001/jama.288.12.1499 